\title{
Herz, Birgit
}

\section{Professionalität in pädagogischen Zwangskontexten. Eine Annäherung} aus der Perspektive der Pädagogik bei Verhaltensstörungen

Emotionale und soziale Entwicklung in der Pädagogik der Erziehungshilfe und bei Verhaltensstörungen : ESE 3 (2021) 3, S. 78-87

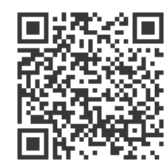

Quellenangabe/ Reference:

Herz, Birgit: Professionalität in pädagogischen Zwangskontexten. Eine Annäherung aus der Perspektive der Pädagogik bei Verhaltensstörungen - In: Emotionale und soziale Entwicklung in der Pädagogik der Erziehungshilfe und bei Verhaltensstörungen : ESE 3 (2021) 3, S. 78-87 - URN: urn:nbn:de:0111-pedocs-225981 - DOI: 10.25656/01:22598

https://nbn-resolving.org/urn:nbn:de:0111-pedocs-225981

https://doi.org/10.25656/01:22598

in Kooperation mit / in cooperation with:

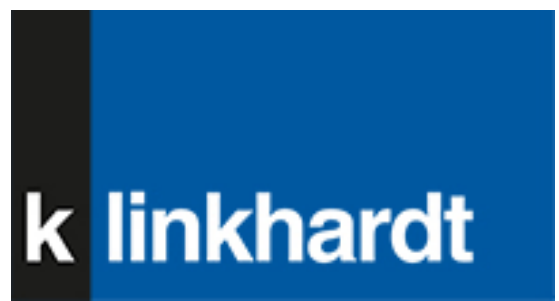

http://www.klinkhardt.de

\section{Nutzungsbedingungen}

Dieses Dokument steht unter folgender Creative Commons-Lizenz: http://creativecommons.org/licenses/by-nc-nd/4.0/deed.de - Sie dürfen das Werk bzw. den Inhalt unter folgenden Bedingungen vervielfältigen, verbreiten und öffentlich zugänglich machen: Sie müssen den Namen des Autors/Rechteinhabers in der von ihm festgelegten Weise nennen. Dieses Werk bzw. dieser Inhalt darf nicht für kommerzielle Zwecke verwendet werden und es darf nicht bearbeitet, abgewandelt oder in anderer Weise verändert werden.

Mit der Verwendung dieses Dokuments erkennen Sie die Nutzungsbedingungen an.

\section{Terms of use}

This document is published under following Creative Commons-License: http://creativecommons.org/licenses/by-nc-nd/4.0/deed.en - You may copy, distribute and transmit, adapt or exhibit the work in the public as long as you attribute the work in the manner specified by the author or licensor. You are not allowed to make commercial use of the work or its contents. You are not allowed to alter, transform, or change this work in any other way.

By using this particular document, you accept the above-stated conditions of use.

\section{Kontakt / Contact:}

\section{peDOCS}

DIPF | Leibniz-Institut für Bildungsforschung und Bildungsinformation Informationszentrum (IZ) Bildung

E-Mail: pedocs@dipf.de

Internet: www.pedocs.de

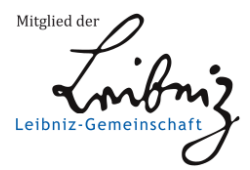



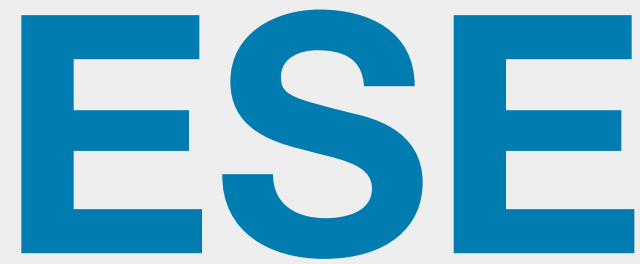

\section{Emotionale und Soziale}

Entwicklung in der Pädagogik der Erziehungshilfe und bei Verhaltensstörungen

\section{Heft 3}

Kompetent im NETZwerk:

Realität - Illusion - Vision?!

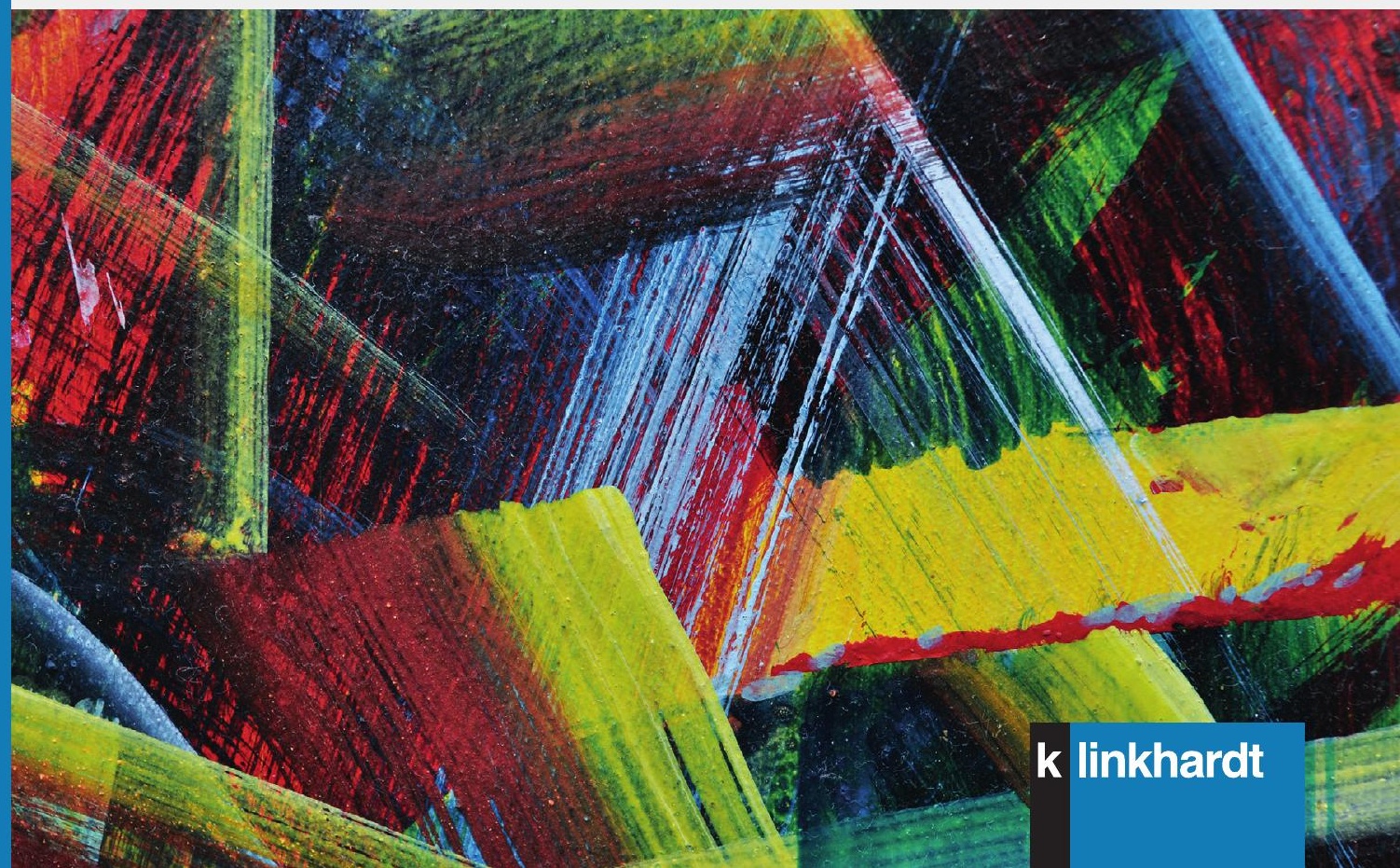




\title{
ESE
}

\author{
Emotionale und Soziale Entwicklung \\ in der Pädagogik der Erziehungshilfe und \\ bei Verhaltensstörungen
}




\section{ESE}

Emotionale und Soziale Entwicklung

in der Pädagogik der Erziehungshilfe und

bei Verhaltensstörungen

\section{Herausgebende Redaktion}

Stephan Gingelmaier (Sprecher der Redaktion)

Werner Bleher

Birgit Herz

Janet Langer

Lars Dietrich

Reinhard Markowetz

\section{Redaktionelle Unterstützung}

Anna Beyer 


\section{ESE}

Emotionale und Soziale Entwicklung in der Pädagogik der Erziehungshilfe und bei Verhaltensstörungen

3. Jahrgang (2021)

Heft 3

Kompetent im NETZwerk: Realität - Illusion - Vision?! 
Die Open Access-Publikation der Zeitschrift ESE wurde von verschiedenen Lehrstüblen und Personen der Dozierendenkonferenz der Forschenden und Lehrenden der „Pädagogik bei Verhaltensstörungen”। des Förderschwerpunkts „emotionale und soziale Entwicklung” finanziert.

Korrespondenzadresse:

Prof. Dr. Stephan Gingelmaier

Psychologie und Diagnostik im Förderschwerpunkt Emotionale und Soziale Entwicklung

PH Ludwigsburg

Reuteallee 46

71634 Ludwigsburg

Erscheinungsweise:

ESE Emotionale und Soziale Entwicklung in der Pädagogik der Erziehungshilfe und bei Verhaltensstörungen erscheint jährlich, jeweils im Sommer.

Die Hefte sind über den Buchhandel zu beziehen.

Das Einzelheft kostet EUR (D) 24,90, im Abonnement EUR (D) 24,90 (ggfs. zzgl. Versandkosten).

Das Abonnement für Studierende kostet EUR (D) 19,90 (ggfs. zzgl. Versandkosten)

nur bei Vorlage einer aktuellen Immatrikulationsbescheinigung.

Bestellungen und Abonnentenbetreuung:

Verlag Julius Klinkhardt

Ramsauer Weg 5

D-83670 Bad Heilbrunn

Tel: +49 (0)8046-9304

Fax: +49 (0)8046-9306

oder nutzen Sie unseren webshop:

www.klinkhardt.de

Bibliografische Information der Deutschen Nationalbibliothek

Die Deutsche Nationalbibliothek verzeichnet diese Publikation in der Deutschen Nationalbibliografie; detaillierte bibliografische Daten sind im Internet abrufbar über http://dnb.d-nb.de.

2021.1. () by Julius Klinkhardt.

Bildnachweis Umschlagseite 1: ( ) Petr Hrbek, 1992, Ohne Titel (Ausschnitt);

mit freundlicher Genehmigung von Ursula Binder und Martina Hoanzl.

Druck und Bindung: AZ Druck und Datentechnik, Kempten.

Printed in Germany 2021.

Gedruckt auf chlorfrei gebleichtem alterungsbeständigem Papier.

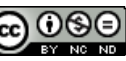

Die Publikation (mit Ausnahme aller Fotos, Grafiken und Abbildungen) ist veröffentlicht unter der Creative Commons-Lizenz: CC BY-NC-ND 4.0 International

https://creativecommons.org/licenses/by-nc-nd/4.0/

ISBN 978-3-7815-5903-5 Digital

doi.org/10.35468/5903

ISBN 978-3-7815-2467-5 Print

ISSN 2629-0170 


\section{Inhaltsverzeichnis}

Editorial der Herausgebenden 7

I Originalia

Perspektiven von Bediensteten des Jugendstrafvollzugs auf pädagogische Beziehung tiefenhermeneutische Einsichten aus einer qualitativ-empirischen Studie Janet Langer, Pierre-Carl Link, Ulrike Fickler-Stang und David Zimmermann

Erfassung der Lehrer*innen-Schüler*innen-Beziehung - ein Überblick über Erhebungsverfahren und -instrumente für die (sonder-)pädagogische Forschung und Praxis

Tatjana Leidig, Tijs Bolz, Émilie Niemeier, Jannik Nitz und Gino Casale

Multiprofessionalität und Netzwerke als Generallösung? -

Ein Plädoyer für einen Perspektivwechsel

Andrea Bethge

Mentalisieren im Netzwerk? Das Adaptive Mentalization-Based Integrative Treatment (AMBIT) als (inter-)professioneller Ansatz im Kontext der Pädagogik bei Verhaltensstörungen Andrea Dlugosch

Professionalität in pädagogischen Zwangskontexten:

Eine Annäherung aus der Perspektive der Pädagogik bei Verhaltensstörungen

Birgit Herz. .78

Professionalisierung im Kontext externalisierender Verhaltensprobleme -

Entwicklung eines Qualifizierungs- und Begleitkonzepts für Lehrkräfte an Förderschulen mit dem Förderschwerpunkt Emotionale und soziale Entwicklung

Tatjana Leidig, Charlotte Hanisch, Ulrike Vögele, Émilie Niemeier, Silke Gerlach

und Thomas Hennemann

Kunstunterricht im Kontext von Unterrichts- und Verhaltensstörungen -

Zur Konzeption eines didaktischen Planungsmodells für Kunst- und

Sonderpädagoginnen und -pädagogen

Daniel Ricci.....

Netzwerk B ${ }^{2}$ : Betrieb und Berufsschule in der inklusiven Ausbildungsvorbereitung eine Fragebogenstudie zu zentralen Bedingungs- und Wirkfaktoren

Francesco Ciociola, Stefanie Roos und Christoph de Oliveira Käppler.

aRT - ein Ansatz zur wissenschaftlich gestützten Schulberatung Janna Rühl, Pascal Schreier, Stephanie Blatz und Roland Stein 
Praxis-Forschungs-Netzwerke am Beispiel des Projekts „Geschwisterklassen“

Désirée Laubenstein und David Scheer

III Praxis und Theorie

Netzwerkarbeit im Kinderschutz. Visionen für die Kooperation bei Gefährdung des Wohls von sonderpädagogisch markierten Kindern

Susanne Leitner.

Umsetzung und Evaluation eines multiprofessionellen und systemübergreifenden Inklusionskonzeptes für Kinder und Jugendliche im Schulalter Karolina Urton, Sophia Hertel und Thomas Hennemann

IV Buchbesprechungen

Das eigensinnige Kind. Über unterdrückten Widerstand und die Formen ungelebten Lebens - ein gesellschaftspolitischer Essay

Pierre-Carl Link

V Forum: Kurzberichte aus den Bundesländern

Bayern: Neuer Standort der Pädagogik bei Verhaltensstörungen in Regensburg

Bernhard Rauh, Pierre-Carl Link und Philipp Abelein

VI Neues aus dem Fach (Zeitraum 2020 - Friuhjahrr 2021) 
Professionalität in pädagogischen Zwangskontexten: Eine Annäherung aus der Perspektive der Pädagogik bei Verhaltensstörungen

Birgit Herz 


\section{Abstract}

Extreme psychische und physische Belastungen aus der Primärsozialisation von überwiegend männlichen Heranwachsenden kennzeichnen die Lebenslagen der Inhaftierten. Im Jugendstrafvollzug konzentriert sich eine vielschichtige Gewaltakkumulation, die durch die massiven Viktimisierungserfahrungen ein hohes Potential an destruktiver Gruppendynamik entfaltet. Ein juridisch normierter Rahmen in dieser "geschlossenen Institution“ begrenzt Bildung und Erziehung und verweist sie in einen pädagogischen Zwangskontext. Der Beitrag versucht, eine theorie- wie praxisorientierte Annäherung an pädagogische Professionalität in Zwangskontexten wie im Jugendstrafvollzug zu leisten.

\section{Keywords}

Jugendstrafvollzug, Viktimisierungserfahrungen männlicher Inhaftierter, Pädagogik in Zwangskontexten 


\section{Einleitung}

Im Jahr 2007 veröffentlichten Franziska Lamott und Martin Schott eine Analyse der destruktiven Dynamik von Gruppenprozessen. Fallmaterial lieferte eine deutsche Jugendstrafanstalt, in der die Gruppendynamik für ein Gruppenmitglied zur Ermordung führte (Lamott $\&$ Schott, 2007). Lamott und Schott reflektieren mittels psychoanalytischer Forschungsbefunde die Konsequenzen einer Jungensozialisation im Kontext dominanter männlicher (väterlicher) Gewalt und die hieraus resultierenden psychodynamischen Konsequenzen für das Leben unter Haftbedingungen. 2018 begingen zwei Jugendliche in der schottischen Jugendstrafanstalt Pulmont Selbstmord. Diese Vorfälle waren der Anlass für eine ausführliche Bestandsaufnahme über die Situation in diesem Gefängnis und sie führten zu umfassenden Empfehlungen einer Expertenkommission an die schottische Regierung (Sinclair-Gieben, 2019). Selbstmorde in Jugendstrafanstalten sind kein singuläres Phänomen, und sie geben auch international immer wieder begründete Anlässe für eine dezidierte Kritik am Disziplinar- und Zwangssystem freiheitsentziehender Maßnahmen im Hinblick auf mögliche destruktive Sekundäreffekte für inhaftierte Kinder und Jugendliche (Radeloff et al., 2015; Fazel, Ramisch \& Hawton, 2017).

Mord und Selbstmord dokumentieren im Extrem jene drei Kerndimensionen, die für eine erziehungswissenschaftliche Annäherung an die praxisbezogenen pädagogischen Herausforderungen des Jugendstrafvollzugs von besonderer Relevanz sind und zugleich auf dilemmatische und hochkomplexe Rahmenbedingungen verweisen:

1. Extreme psychische und physische Belastungen aus der Primärsozialisation kennzeichnen die Lebenslagen der Inhaftierten.

2. Im Jugendstrafvollzug konzentriert sich eine vielschichtige Gewaltakkumulation, bei der männliche Geschlechterrollen dominieren.

3. Ein juridisch normierter Rahmen begrenzt Bildung und Erziehung und verweist sie in einen pädagogischen Zwangskontext.

Im Positionspapier von Forschenden und Lehrenden in der ,Pädagogik bei Verhaltensstörungen '/des Förderschwerpunktes, emotionale und soziale Entwicklung' an bundesdeutschen Hochschulen steht auch der Jugendstrafvollzug im Fokus der theorie- wie praxisbezogenen Expertise (Bleher \& Gingelmaier, 2019):

Außerschulische Arbeitsfelder mit dem inhaltlichen Schwerpunkt der Pädagogik bei Verhaltensstörungen finden sich ebenso im Bereich der ambulanten wie auch der stationären Maßnahmen des Jugendstrafrechts im Jugendarrest und Jugendstrafeinrichtungen, auch im Erwachsenenvollzug und in Projektalternativen zum Jugendstrafvollzug (Bleher \& Gingelmaier, 2019, S. 100).

Die Vertreter/innen des Faches bemühen sich originär um die Erhaltung als förderlich erkannter guter und um die Verbesserung als problematisch anzusehender Aufwuchs-, Lebens-, Lern- und Teilhabebedingungen aller jungen Menschen unter 27 Jahren. [...] Nach den Erkenntnissen der Trauma-, Bindungs-, Risiko- und Resilienzforschung weisen vor allem junge Menschen, deren Entwicklung in psychosozialen Problemlagen und dysfunktionalen Erziehungsmilieus - nicht nur ökonomisch prekär - stattfindet, ein hohes Risiko auf, mit ihrem emotionalen Erleben und sozialen Handeln ausgegrenzt zu werden, auf Unverständnis und Ablehnung zu stoßen und ggf. auch sanktioniert oder sogar strafrechtlich verfolgt zu werden. (Bleher \& Gingelmaier, 2019, S. 95).

Dieser Beitrag versucht, eine theorie- wie praxisorientierte Annäherung an pädagogische Professionalität in Zwangskontexten wie im Jugendstrafvollzug zu leisten. Dabei sind zunächst einige Zahlen, Daten und Fakten zu benennen, um das Feld dieses pädagogischen Zwangs- 
kontextes zu konkretisieren und vor dem Hintergrund der je individuellen Biographien der Inhaftierten verallgemeinerbare Kernaussagen zusammenzufassen, so dass erste Ansätze und Konturen einer pädagogischen Professionalität skizziert werden können.

Zugleich muss die Dialektik zwischen Zwang und Erziehungsauftrag im Jugendstrafvollzug samt ihrer Produktion und Reproduktion von Macht- und Ohnmachtdynamiken berücksichtigt werden, um in Anlehnung an Bettinger „die Eigenaktivitäten und Eigensinnigkeiten der Subjekte als Bearbeitungsformen von gesellschaftlichen oder institutionellen Ausschließungserfahrungen und damit als Teil der Konflikte um gesellschaftliche Positionierungen zu fassen“ (Bettinger, 2015, S. 172). Die Komplexität manifest normsetzender Prozesse erfordert zwangsläufig, den theoretischen Zugang zum Untersuchungsfeld Jugendstrafvollzug mit der Spezifizierung und Transparenz der eigenen wissenschaftlichen Perspektive auszuweisen. Der vorliegende Beitrag orientiert sich an der Psychoanalytischen Pädagogik (Gerspach, Eggert-Schmid-Noerr, Naumann \& Niederreiter, 2014) sowie an der kritischen Erziehungswissenschaft (Walgenbach, 2019), um einen Theorie- und Praxistransfer aus der Perspektive der Pädagogik bei Verhaltensstörungen zu herzustellen.

\section{Die Klientel im Jugendstrafvollzug: Zahlen, Daten, Fakten}

Derzeit sinkt national wie international die Inhaftierungsrate im Jugendstrafvollzug (Dünkel, 2018; Baier, 2019). Dünkel, Geng und Harrendorf mahnen allerdings 2019: „Der Belegungsrückgang sollte nicht als Möglichkeit gesehen werden, die grundsätzliche relativ gute personelle Ausstattung zu reduzieren, sondern als Chance für die Entwicklung eines qualitativ verbesserten Vollzugs verstanden werden“ (Dünkel, Geng \& Harrendorf, 2019, S. 328). Diese rückläufige Kriminalitätsentwicklung steht in deutlichem Gegensatz zur medialen Berichterstattung, öffentlichen Wahrnehmung und Diskussion (Dünkel, Geng \& Harrendorf, 2019).

Insgesamt befanden sich 2018 in den Jugendstrafvollzugsanstalten der Bundesrepublik Deutschland 3701 Insassen. Die Geschlechterverteilung zeigt mit 96,1\% männlicher Verurteilter eine deutliche Überrepräsentanz von heranwachsenden Männern. Ihre faktische Inhaftierung beträgt durchschnittlich ca. eineinhalb Jahre. Die Verteilung auf die einzelnen Bundesländer divergiert allerdings erheblich: Bei einer durchschnittlichen Gefangenenrate von 55 Gefangenen auf 100000 Einwohnerinnen und Einwohner liegt die Rate in den Bundesländern Bremen mit 21,9 und Hamburg mit 35,5 Inhaftierten deutlich unter dem Bundesdurchschnitt. Bayern mit 61,1 und Berlin mit 67,8 weisen demgegenüber die höchsten Quoten auf (Dünkel, Geng \& Harrendorf, 2019).

In Bezug auf die Bildungsabschlüsse von Inhaftierten konnten für die Jugendstrafanstalt Hameln in Niedersachsen bspw. anhand der Fallzahlen zwischen 2009 und 2013 Entwicklungstendenzen bei den Bildungsabschlüssen aufgezeigt werden. Klebe (2016) fasst seine diesbezüglichen empirischen Untersuchungen folgendermaßen zusammen:

Entgegen der Annahme, dass arbeitsmarktrelevante Schulabschlüsse eine unmittelbare, präventive Wirkung auf Delinquenz und Inhaftierung ausüben würden, stieg der Anteil der inhaftierten Jugendlichen, die einen Haupt- oder Realschulabschlussabschluss absolviert hatten im Zeitraum 2009-2013 deutlich an (von 33\% auf 41\%), während der Anteil der Inhaftierten ohne arbeitsmarktrelevanten Abschluss von 66\% auf 59\% sank (Klebe, 2016, S. 3). 
Hieraus könnte - unter Vorbehalt und eher spekulativ - die These formuliert werden, dass sich die generelle arbeitsmarktpolitische Abwertung von Schulabschlüssen auch nachteilig auf die Legalbewährung der Inhaftierten auswirkt. Als ein weiterer Aspekt könnte sich möglicherweise aus der generellen Zunahme und Aufwertung sogenannter höherer Bildungsabschlüsse ergeben.

Im internationalen Vergleich ist besonders hervorzuheben, dass der Beginn des Strafmündigkeitsalters in Europa bspw. von 10 Jahren in Irland bis zu 15 Jahren in Griechenland variiert und in Lateinamerika zwischen dem 12. und 14. Lebensjahr liegt. In muslimischen Ländern können bereits 7-jährige Kinder strafmündig sein, vergleichbares gilt auch für viele Staaten der USA (Dünkel, 2018).

Die Strafrechtsverfolgung im Kindesalter ab dem zehnten Lebensjahr (bspw. auch in England, Schottland und Wales) betrifft eine Gruppe von ganz jungen Menschen, die sich in einer ganz besonderen biopsychosozialen Entwicklungs- und Lebensphase befinden. Der normdurchsetzende Eingriff geht hier einher mit der kognitiven, emotionalen und sozialen Verarbeitung eines temporären gesellschaftlichen Ausschlusses. Diese besondere Form der Exklusion spitzt zugleich bereits bestehende materielle und kulturelle Armut zu und verschärft ohnehin psychisch hoch belastete (und belastende) Autonomiekrisen (Herz, 2013a; Herz, 2017). Inhaftierungen im Jugendalter, einer hochriskanten Entwicklungsphase (Schwarzer \& Gingelmaier, 2020), verstärken bereits bestehende Vulnerabilitätskrisen. Die ,Adoleszenz als zweite Chance‘ erhält hier keine Möglichkeit zur Realisierung eines Perspektivenwechsels (Eisler, 1966; Erdheim, 1996).

Nationale und internationale Forschungsbefunde belegen nicht nur eine drastische Dominanz des männlichen Geschlechts bei den Inhaftierten, sondern auch die augenfällige Prävalenz ihrer massiven familiären Gewalterfahrungen und vielfach dysfunktionalen Familiensysteme innerhalb spezifischer Milieus (Baier, Kamenowski, Manzoni \& Haymoz, 2019; SinclairGieben, 2019). Die Viktimisierungsbelastung bildet die bedeutsamste Gemeinsamkeit unter den inhaftierten Kindern und Jugendlichen. Normverletzendes Verhalten ist oftmals das zumeist ungeplante Resultat einer individuellen Konfliktlösung. Dieser Modus stabilisiert sich allerdings insbesondere dann, wenn ausreichende psychosoziale und materielle Ressourcen längst nicht mehr zur Verfügung stehen, um dauerhafte oder aktuelle individuelle, gruppenund/oder institutionenbezogene Konflikte mit gesellschaftlich legitimen Handlungsroutinen zu lösen (Herz, 2006; Herz, 2013a).

Diese Heranwachsenden betreten die ,Bühnen' des Jugendstrafvollzugs mit einem hohen Potential destruktiver Dynamiken, die aus schwerem Ohnmachts-, Hilflosigkeit- und Angsterleben resultieren. Deren (Moral- und Gesetzesregeln ignorierende) destruktive ,Übersetzungen' in normverletzendes Verhalten mit gruppenspezifischen Deliktinszenierungen werden im Gefängnis freilich nicht pädagogisch-psychoanalytisch, sondern mit juridisch legitimiertem Arsenal an disziplinierenden rigiden Routinen der Normdurchsetzung sanktioniert. Gehorsamkeitsdrill auf der Grundlage des Status als ,Unfreier' während der Zeit ihres Aufenthaltes im Jugendstrafvollzug soll ihre Legalbewährung nach der Haftentlassung stärken und vorbereiten.

Schrapper (2015) differenziert zwischen drei unterschiedlich konnotierten Funktionen von Strafe: Einerseits repräsentiert sie eine deutlich sozial wahrnehmbare Konsequenz auf Normverletzungen, ist anderseits eine routinierte Strategie zur Abschreckung vor Wiederholungstaten und sie dient dem Ausgleich des durch die Tat verursachten Schadens (Schrapper, 2015). Schon diese knappen Kennzeichnungen weisen dem Jugendstrafvollzug eine Multifunktio- 
nalität zu, deren Kernaufgabe die Gegensteuerung von bereits über Jahre hinweg sich verfestigenden Überlebensstrategien in komplexen Risiko- und Belastungslagen ist. Er soll allerdings zugleich auch das Scheitern und Versagen vorgelagerter Institutionen der öffentlichen Bildung und Erziehung ,nachbessern“ - also das, was ein Jugendrichter „Nachreifung in der Haft" nennt (Hoyer, 2020, S. 119).

Der Jugendstrafvollzug repräsentiert faktisch und auf symbolischer Ebene das Scheitern der der Inhaftierung vorgelagerten Institutionen der primären und sekundären Sozialisation, die in unterschiedlichen Stufen, Phasen, Graden und Dynamiken an den Prozessen des Ein- und Ausschließens beteiligt sind (Homann, 2019). „This weight of evidence poses fundamental questions about the failure of education, health and family support services" (Hughes \& Pierse O’Bryne, 2016, S. 15). Allerdings geht mit den veränderten Etikettierungen - kriminell, delinquent, u.ä.m. - eine interinstitutionelle Verantwortungsdelegation einher. Diese neuen Etiketten „verdecken damit auch die Konflikte, die die Institutionen bei ihrer Platzierungsarbeit darüber erzeugen, dass institutionell diskriminierte und vielfach selektierte Jugendliche, die in chancenverschließende Karrieren gedrängt werden, sich mit diesen Prozessen der Degradierung und Ausschließung als Konfliktsubjekte auseinandersetzen müssen“" (Stehr, 2014, S. 44).

Mit dem Wechsel aus dem System der Kinder- und Jugendhilfe bspw. in das strafverfolgende System wechselt auch der Status der betroffenen Jugendlichen: „... once in the criminal justice system their previous status as vulnerable and in need of support, becomes renounced for that of the risk-posing offender requiring management or containment" (Fitzpatrick, Williams \& Coyne, 2016, S. 8). Hier übernimmt dann der Jugendstrafvollzug die Rolle einer „als-ob-Konstruktion“, gleichsam „als-ob“ er jetzt die erwünschten normativen Anforderungen an die Verhaltenskorrekturen seiner Inhaftierten einzulösen in der Lage sei. Diese Pseudokonstruktion, zumeist übersetzt in wohlfeilem Resozialisierungsdeutsch, suggerieren eine Versprechung, die selbst wiederum zu neuen Enttäuschungen und Belastungen führt.

Diesem unreflektierten Anspruch mit seinen hoch wirkmächtigen Omnipotenzphantasien wird die Institution Jugendstrafanstalt nicht gerecht, was sowohl die Rückfallquoten als auch die gelingende Legalbewährung als gesellschaftliche Normparameter betreffen (Dünkel, 2018). So schreiben bspw. Hoops und Holthusen (2015), dass ein konzeptionell ausgereiftes Übergangsmanagement durch Besonderheit und Rarität gekennzeichnet ist, da zwar Projekte an den Schnittstellen zwischen Vollzugsanstalt und Institutionen der Resozialisierung bestehen, die allerdings weder flächendeckend noch mit gesicherten Ressourcen anzutreffen seien (Hoops \& Holthusen, 2015). Das implizite Versprechen, nach dem Scheitern bisheriger familiärer, schulischer und außerschulischer Hilfe- und Unterstützungssysteme, Normalisierungsprozesse im dominanzkulturellen Verständnis von Verhaltenskorrekturen zu initiieren, zu begleiten und zu stabilisieren, löst der Jugendstrafvollzug nämlich auch nicht ein, worauf Neubacher und Schmidt (2018) deutlich aufmerksam machen:

„In Anbetracht des Umstandes, dass das Ziel des Strafvollzugs die Legalbewährung ist, d.h. die Rückfallvermeidung, können die Befunde der Rückfallforschung nicht zufriedenstellen Mit rund 70\% Rückfälligen im Zeitraum von drei Jahren nach Entlassung (bzw. 80\% nach sechs Jahren) hat der Strafvollzug - verglichen mit anderen Sanktionen - die höchste Rückfallquote“ (Neubacher \& Schmidt, 2018, S. 770). 


\section{Einige Überlegungen über die Psychodynamik von Gewalt im Jugendstrafvollzug}

Mit der Kombination aus traumatisierender Sozialisation und (qua Scheitern) entwicklungsdestabilisierender öffentlicher Bildung und Erziehung verstärkt der Status als Strafgefangener im Jugendgefängnis eine Intensivierung bereits bestehender destruktiver Dispositionen. „Wer Opfer wird, tritt später mit höherer Wahrscheinlichkeit als Täter hervor“ (Neubacher \& Schmidt, 2018, S. 773). McCarthy, Schiraldi und Shark (2016) stellen fest:

The trauma many of these young people have experienced makes them especially sensitive to environmental triggers, and yet, many are kept in institutional environments that seem designed to trigger trauma and rage: long periods of isolation; harsh, sterile surroundings; bright light; a constant dim; and a near-constant threat of violence (McCarthy, Schiraldi \& Shark, 2016, S. 5; Shalev, 2018).

Auch wenn im Vergleich zu dieser Kritik am US-amerikanischen Jugendstrafvollzug die äußeren Rahmenbedingungen in der Bundesrepublik Deutschland nicht ganz so dramatisch erscheinen mögen, erzeugt das System spezifische Dynamiken. Frank Neubacher und Holger Schmidt belegen, dass in den 27 Jugendstrafanstalten in Deutschland insbesondere Gewalt unter den Strafgefangenen ubiquitär ist.

Verknüpft man die in Haft ausgeübte Gewalt mit der vorinstitutionellen Biographie der jungen Männer, so zeigt sich, dass vor allem die im familiären Kontext erlittenen Ohnmachts- und Missachtungserfahrungen von Bedeutung sind. [...] Die hierarchische Gefangenensubkultur scheint dabei besonders von jenen Gefangenen als anerkennungsstiftendes Identitätsangebot wahrgenommen zu werden, die wiederholte und schwere innerfamiliäre Viktimisierungen erlitten hatten (Neubacher \& Schmidt, 2019, S. 774).

Internationale Studien belegen insbesondere den Zusammenhang zwischen psychischer Strukturbildung aufgrund massiver Traumatisierungen durch belastende Risikobiographien und deren destruktive Potentialentfaltung unter den Bedingungen der Inhaftierung (Ford, Chapmann \& Connor, 2012; Moore, Gaskin \& Indig, 2013; Hughes et al., 2015). Dieser Konnex skandalisiert das nach wie vor bestehende dehumanisierende Versagen öffentlicher Bildungs- und Erziehungsinstitutionen im Kinderschutz (Bode \& Turba, 2015). Kläsener und Ziegler (2020) stellen hier - durchaus provokant - fest, dass die Kategorie Kindeswohlgefährdung mit der Kategorie der Leistungsberechtigung, „die weniger mit wohlfahrtsstaatlichen Rechten als mit Sittlichkeitsnormen der Regulierung von Familien seit dem Kaiserreich zu (tun, B.H.) hat" (Kläsener \& Ziegler, 2020, S. 36). Im Verbund mit der prekären Ressourcenlage in den Kommunalen Sozialdiensten (Beckmann, Ehlting \& Klaes, 2018) entsprechen die aufgrund von Kindeswohlgefährdung veranlassten Kinder- und Jugendhilfemaßnahmen dem professionsspezifischen Fachwissen nur eingeschränkt. Es wird von politischer Seite aus billigend in Kauf genommen, dass intervenierende Maßnahmen scheitern und ein Kreislauf an möglichen Folgemaßnahmen beginnt (Herz, 2020).

Insofern illustrieren die Ergebnisse der aktuellen retrospektiven Studie von Lunz (2019) über geschlossene Jugendhilfeeinrichtungen in Luxemburg (vergleichbar mit deutschen Jugendstrafanstalten) genau jene destruktiven Übersetzungs'leistungen' ehemals viktimisierter Inhaftierter: „Ritualisierte Praktiken von Gewalt und machtvolle, körperliche Inszenierungen sowie verschiedene Formen von Intrigen untereinander werden zur Herstellung und 
Beibehaltung von Rangordnungen innerhalb der Peers genutzt“ (Lunz, 2019, S. 90). Eine Spezifizierung der Gewaltpraktiken ist insbesondere die sexuelle Gewalt im Jugendstrafvollzug (Neubacher \& Schmidt, 2017).

Diese Rangordnungen werden maßgeblich unbewusst dynamisiert durch die verdrängten und abgespaltenen Ängste der durch Ohnmacht und Hilflosigkeit gekennzeichneten biographischen Erfahrungen katastrophaler Einsamkeit bei Viktimisierungen (Bohleber, 2006). Diese scheinen oft nur über eine Identifikation mit Männlichkeitsmythen erträglich zu werden, da sie eine unbewusste Kompensationsstrategie eröffnen. Baier u.a. sprechen hier in Bezug auf den Jugendstrafvollzug explizit von „toxischer Männlichkeit“ (Baier et al., 2019).

\section{Pädagogische Professionalität in Zwangskontexten}

Die Pädagogik bei Verhaltensstörungen verfügt über ein fundiertes Expertenwissen in Bezug auf traumatisierte Kinder und Jugendliche (Zimmermann, 2015a; 2015b), in Bezug auf eine gelingende entwicklungsorientierte schulische ebenso wie außerschulische Pädagogik (Stein \& Müller, 2016) im Hinblick auf interprofessionelle Kooperation von Institutionen der sekundären Sozialisation und deren Kritik (Herz, 2017; Herz, 2020). Es käme allerdings einer Verdrängung gleich, dieses Expert*innenwissen ohne kritisch-distanzierte Reflexionen über erziehungsstrafrechtliche, und damit freiheitsentziehende Maßnahmen auf das spezifische Setting ,Jugendstrafvollzug' transferieren zu wollen.

Eine pädagogische Professionalität müsste hier zunächst einen geschützten Rahmen ermöglichen, aus der Subjektperspektive aller Personen im Jugendstrafvollzug die tabuisierten emotionalen Dynamiken aus Angst und Angstabwehr und damit Macht- und Ohnmachtsgefühle zu reflektieren und zugleich die latent und manifest damit verknüpften institutionell/ organisationalen Abläufe, wie bspw. Hierarchien, Zeitmanagement, Besoldungsregelungen etc. in den Blick zu nehmen. Dies wäre als ,äußerer Rahmen' der Aufgabenbeschreibung angemessene Modellüberlegungen über pädagogische Professionalität in Zwangskontexten zu bedenken, um bspw. auch die disziplinierende Dimension des, Wartens ${ }^{\text {` }}$ - und damit der Funktionalisierung von Zeit als ein zentraler Faktor der strafritualisierten Zwangskontexte (Foucault, 1993) - als „Hauptstück in der Dramaturgie des Arrests und der Inhaftierung“ zu reflektieren (Eckold, 2019, S. 329).

Die Dialektik zwischen dem eingangs erwähnten berufsfeldspezifischen, empirisch fundierten Wissen und den konkreten Praxisbedingungen pädagogischer Zwangskontexte - die ja auch die Ressourcensituation im Jugendstrafvollzug berücksichtigen muss - offenbart in aller Deutlichkeit das Versagen der nach wie vor bestehenden „obrigkeitsstaatlichen Fürsorgetradition" (Kläsener \& Ziegler, 2018, S. 37). Pädagogische Fachkräfte im Jugendstrafvollzug sind zunächst zuvorderst Mitglieder einer spezifischen exkludierenden Institution, die qua öffentlichem Auftrag mit dem Scheitern der zuvor auf Inklusion hin vorlagerten Institutionen der - ebenfalls öffentlichen - Bildungs- und Erziehungsinstitutionen konfrontiert ist. Im Hinblick auf die rückläufigen Inhaftierungszahlen, der weiterhin bestehenden Infrastruktur und den professionellen Anforderungen an Fachkräfte in pädagogischen Zwangskontexten gilt es, aus der Perspektive der Pädagogik bei Verhaltensstörungen innerhalb des Jugendstrafvollzugssystems selbst für hierauf bezogene Verbesserungen zu plädieren. Hier sind zielgruppenspezifische motivationale Einstellungen und Haltungen ebenso bedeutsam wie versiertes Fachwissen. Hinsichtlich der gewaltaffinen Selbstinszenierungen der Inhaftierten erhalten genau jene Kernkompetenzen, wie sie bspw. die Psychoanalytische Pädagogik betont, 
einen besonderen Stellenwert. Hier ist insbesondere die Fähigkeit zur professionellen Selbstreflexion und zur sachlich-neutralen Distanzierung ebenso wie zur authentischen Teilhabe im Kontext von Scham und Beschämung, Verzweiflung und Dreistigkeit, physischer Kraft und psychischer Hilfsbedürftigkeit - um nur einige der widerstreitenden Ambivalenzkonflikte zu erwähnen - besonders hervorzuheben (Herz, 2013b). Eine derartige Neuausrichtung bräuchte zudem eine stärkere Verzahnung jener interprofessionellen Kooperationsnetzwerke, die der Inhaftierung vorgelagert sind. Infolgedessen müssten bspw. auch Team- und Leitungskompetenzen als zu implementierende professionelle Relevanzbereiche im Feld Jugendstrafvollzug unterstützt und gefördert werden, um für die destruktiven Gruppendynamiken innerhalb horizontaler und vertikaler Hierarchien bewusste offene und enttabuisierte Reflexionsräume zu schaffen.

\section{Literatur}

Baier, D. (2019). Jugendkriminalität in der Schweiz: Entwicklung und Einflussfaktoren. Zeitschrift für Jugendkriminalrecht und Jugendhilfe, 30(3), 214-223.

Baier, D., Kamenowski, M., Manzoni, P. \& Haymoz, S. (2019). „Toxische Männlichkeit“ - die Folgen gewaltlegitimierender Männlichkeitsnormen für Einstellungen und Verhaltensweisen. Kriminalistik, 73(7), 465-471.

Beckmann, K., Ehlting T. \& Klaes, S. (2018). Berufliche Realität im Jugendamt: der ASD in strukturellen Zwängen. Berlin: Verlag des Deutschen Vereins für öffentliche und private Fürsorge e.V.

Bettinger, F. (2015). Wider die Unterordnung sozialer Arbeit unter die Logiken des Jugendstrafrechts. In B. Redmann \& M. Hußmann (Hrsg.), Soziale Arbeit im Jugendarrest. Zwischen Erziehung und Strafe (S. 144-181). Weinheim, Basel: Beltz Juventa.

Bleher, W. \& Gingelmaier, S. (2019). Zum Selbstverständnis einer sonderpädagogischen Fachdisziplin: Das Positionspapier der Forschenden und Lehrenden der „Pädagogik bei Verhaltensstörungen“/des Förderschwerpunktes „emotionale und soziale Entwicklung an bundesdeutschen Hochschulen. ESE Emotionale und Soziale Entwicklung in der Pädagogik der Erziehungshilfe und bei Verhaltensstörungen, 1(1), 92-101.

Bode, I \& Turba, H. (2015). Warum wird das „ganz normale Chaos“ zum Problem? Jugendämter als Hybridorganisationen mit Souveränitätsverlust. In M. Apelt \& K. Senge (Hrsg.), Organisation und Unsicherheiten (S. 105121). Wiesbaden: Springer.

Bohleber, W. (2006). Selbstentwicklung, Integration und Desintegration in der Adoleszenz. In M. Leuzinger-Bohleber (Hrsg.), Bindung, Trauma und soziale Gewalt: Psychoanalyse, Sozial- und Neurowissenschaften im Dialog (S. 121-141). Göttingen: Vandenhoeck \& Ruprecht.

Dünkel, F. (2018). Internationale Tendenzen im Umgang mit Jugendkriminalität. In B. Dollinger \& H. SchmidtSemisch (Hrsg.), Handbuch Jugendkriminalität (S. 89-118). Wiesbaden: Springer.

Dünkel, F., Geng, B. \& Harrendorf, S. (2019). Entwicklungsdaten zu Belegung, Öffnung und Merkmalen der Insassenstruktur im Jugendstrafvollzug. Zeitschrift für Jugendkriminalrecht und Jugendhilfe, 4, 311-328.

Eckold, A. (2019). Warten im Arrest. Sozialer Sinn, 20(2), 313-336.

Eissler, K.R. (1966). Bemerkungen zur Technik der psychoanalytischen Behandlung Pubertierender nebst einigen Überlegungen zum Problem der Perversion. Psyche, 20(10/11), 837-872.

Erdheim, M. (1996). Psychoanalyse, Adoleszenz und Nachträglichkeit. In W. Bohleber (Hrsg.), Adoleszenz und Identität (S. 82-102). Stuttgart: Verlag Internationale Psychoanalyse.

Fazel, S., Ramisch, T. \& Hawton, K. (2017). Suicide in prison. The Lancet Psychiatry, 4(12), 946-952.

Fitzpatrick, C., Williams, P. \& Coyne, D. (2016). Supporting looked after children and care leavers in the Criminal Justice System: Emergent themes and strategies for change. Prison Service Journal, 226, 8-13.

Ford, J.D., Chapmann, J., Connor, D.F. \& Cruise, K.R. (2012). Complex Trauma and Aggression in Secure Juvenile Settings. Criminal Justice and Behaviour, 39(6), 694-714.

Foucault, M. (1993). Überwachen und Strafen: Die Geburt des Gefängnisses (1. Aufl.). Frankfurt a.M.: Suhrkamp.

Gespach, M., Eggert-Schmid-Noerr, A., Naumann, T. \& Niederreiter L. (Hrsg.) (2014). Psychoanalyse Lehren und Lernen an der Hochschule. Stuttgart: Kohlhammer.

Herz, B. (2006). Lernort Jugendstrafanstalt. In F. Albrecht, M. Jödecke \& N. Störmer (Hrsg.), Bildung, Lernen und Entwicklung (S. 207-220). Bad Heilbrunn: Klinkhardt.

Herz, B. (2017). Disziplinarerziehung durch Gruppenzwang: Erziehungs- und Bootcamps. In B. Herz (Hrsg.), Gruppen leiten - Eine Einführung für pädagogische Praxisfelder (S. 87-96). Opladen, u.a.: Barbara Budrich.

Herz, B. (2013a). Jugendstrafvollzug. In B. Herz (Hrsg.), Schulische und außerschulische Erziehungshilfe. Ein Werkbuch zu Arbeitsfeldern und Lösungsansätzen (S. 263-264). Bad Heilbrunn: Klinkhardt. 
Herz, B. (2013b). Aggression - Macht - Angst. In B. Herz (Hrsg.), Schulische und außerschulische Erziehungshilfe. Ein Werkbuch zu Arbeitsfeldern und Lösungsansätzen (S. 55-66). Bad Heilbrunn: Klinkhardt.

Herz, B. (2017) Psychiatrie, Schule und Jugendhilfe: Kooperation und Grenzen der Fallarbeit. In Bundesarbeitsgemeinschaft der Kinderschutz-Zentren e.V. (Hrsg.), Psychische Erkrankung und Sucht. Passende Hilfen für betroffene Kinder, Jugendliche und Eltern (S. 37-56). Köln: Kinderschutz-Zentren e.V..

Herz, B. (2020). Intensivpädagogik. Sozialmagazin, 45(11), (im Druck).

Homann, D. (2019). Endstation und Neustart? Flexible Hilfen - Die Arbeit mit den sogenannten "Systemsprengern«. Behindertenpädagogik, 58(4), 369-416.

Hoops, S. \& Holthusen, B. (2015). ...Verantwortung übernehmen! Die Aufgaben und Herausforderungen der Jugendhilfe im Kontext des Jugendarrests. In B. Redmann \& M, Hußmann (Hrsg.), Soziale Arbeit im Jugendarrest. Zwischen Erziehung und Strafe (S. 181-197). Weinheim, Basel: Beltz Juventa.

Hoyer, J. (2020). Implizite Konzepte feldspezifischer Expert*innen zur Jugenddelinquenz in Niedersachsen, unv. Dissertation, LUH.

Hughes, N., Williams, W.H., Chitabesan, P., Walesby, R., Monnce, L.T.A. \& Clasby, B. (2015). The Prevalence of Traumatic Brain Injury among Young Offenders in Custody: A Systematic Review. Journal of Head Trauma Rehabilitation, 30(2), 94-195.

Hughes, N. \& Pierse-O’Bryne, K. (2016). Disabled Inside: neurodevelopmental impairments among young people in custody'. Prison Services Journal, 226, 14-21.

Kläsener, N. \& Ziegler, H. (2020). Das Kindeswohl - eine ,abscheuliche Phase‘. Widersprüche, 38(149), 29-44.

Klebe, M. (2016). Bildungshintergrund und Inhaftierung niedersächsischer Jugendstrafgefangener. Sonderpädagogik in Niedersachsen, 44(1), 2-5.

Lamott, F. \& Schott, M. (2007). Destruktive Gruppenprozesse. Zur Psycho- und Soziodynamik von Gewalt. Gruppenpsychotherapie und Gruppendynamik, 43(4), 294-308.

Lunz, M. (2019). Geschlossene Jugendhilfeeinrichtungen. Empirische Ergebnisse aus der Perspektive betroffener Jugendlicher. Österreichisches Jahrbuch für Soziale Arbeit, 77-99.

McCarthy, P. Schiraldi, V. \& Shark, M. (2016). The future of Youth Justice: A Community-Based Alternative to the Youth Prison Model. New Thinking in Community Corrections Bulletin. NCJ 250142, 1-35.

Moore, E., Gaskin, C. \& Indig, D. (2013). Childhood maltreatment and post-traumatic stress disorder among incarcerated young offenders. Child Abuse \& Neglect, 37(10), 861-870.

Neubacher, F. \& Schmidt, H. (2018). Sexuelle und sexualisierte Gewalt im Jugendstrafvollzug. In A. Retkowski, A. Treibel \& E. Tuider (Hrsg.), Handbuch sexualisierter Gewalt und pädagogischer Kontexte. Theorie, Forschung, Praxis (S. 497-505). Weinheim, Basel: Beltz Juventa.

Neubacher, F. \& Schmidt, H. (2018). Von punitiven Tendenzen, knappen Behandlungsressourcen und der Schwierigkeit, dem Einzelnen gerecht zu werden. In B. Dollinger \& H. Schmidt-Semisch (Hrsg.), Handbuch der Jugendkriminalität (S. 767-786). Wiesbaden: Springer.

Radeloff, D., Lempp, T., Herrmann, E:, Kettner, M., Bennefeld-Kersten, K. \& Freitag, C.M. (2015). National total Survey of German adolescent Suicide in Prison. European Child \& Adolescent Psychiatry, 24(2), 219-225.

Schrapper, C. (2015). „Warum tun junge Menschen nicht, was vernünftig ist?“ Über die Vernunft normenverletzenden Verhaltens Jugendlicher und die Paradoxie von Erziehung und Strafe. In B. Redmann \& M. Hußmann (Hrsg.), Soziale Arbeit im Jugendarrest. Zwischen Erziehung und Strafe (S. 15-22). Weinheim.

Schwarzer, N. \& Gingelmaier, S. (2020). >>No Risk - No Fun!<< Adoleszente Hirnentwicklung - Ein neuropsychologischer Beitrag zum Verständnis von Risikoverhalten bei Jugendlichen. Behindertenpädagogik, 50(2), 154-170.

Shalev, S. (2018). Can any good come out of isolation? Probably not. Prison Service Journal, 236, 11-16.

Sinclair-Gieben, W. (2019). HM Chief Inspector's Annual Report 2018-19. Her Majesty's Inspectorate of Prisons for Scotland (HMIPS). https://www.prisonsinspectoratescotland.gov.uk/publications/hm-chief-inspector-prisonsscotland-annual-report-2018-19. Zugegriffen: 12. 8.2020.

Stehr, P. (2014). Der parasoziale Meinungsführer als Akteur der politischen Willensbildung. Quantitative Prüfung eines Modellentwurfs. In D. Frieß, J. Jax \& A. Michalski (Hrsg.), Sprechen Sie EU? Das kommunikative Versagen einer großen Idee. Beiträge zur 9. Fachtagung des DFPK (S. 219-238). Berlin: Frank \& Timme.

Stein, R. \& Müller, T. (2016). Inklusion im Förderschwerpunkt emotionale und soziale Entwicklung. Stuttgart: Kohlhammer.

Walgenbach, K. (Hrsg.), Bildung und Gesellschaft im 21. Jahrhundert. Zur neoliberalen Neuordnung von Staat, Ökonomie und Privatsphäre. Frankfurt, New York: Campus.

Zimmermann, D. (2015a). Das Leiden der anderen. Beziehungstraumatisierungen und institutionelle Abwehr. In B. Herz, D. Zimmermann \& M. Meyer (Hrsg.), „... und raus bist Du!“(S. 49-65). Bad Heilbrunn, Klinkhardt.

Zimmermann, D. (2015b). Trauma und Traumadiagnostik in der Schule. Sonderpädagogische Förderung heute, $39(3), 308-321$. 